\title{
Modal Characteristics of Planar Transmission Lines With Periodical Perturbations: Their Behaviors in Bound, Stopband, and Radiation Regions
}

\author{
Yu-Chiao Chen, Member, IEEE, Ching-Kuang C. Tzuang, Fellow, IEEE, Tatsuo Itoh, Fellow, IEEE, and \\ Tapan Kumar Sarkar, Fellow, IEEE
}

\begin{abstract}
This paper presents the modal characteristics of one-dimensional periodical transmission lines, including the microstrip with even-symmetric periodical perturbations, the microstrip on an electromagnetic bandgap (EBG) surface without via holes, and the electric-magnetic-electric (EME) microstrip, in the bound, stopband, and radiation regions. The Brillouin diagram, or the so-called $k-\beta$ diagram, is employed to represent the dispersion characteristics of the periodical transmission lines. The matrix-pencil method is applied to analysis the surface currents $J_{s}$ obtained by invoking the method of moment simulation, and the results are verified by the finite-element method analyzes. The complex modes in the form of $+j \alpha \pm \beta$ and $-j \alpha \pm \beta$ could be observed in the microstrip with even-symmetric periodical structure and form the stopband of the periodical microstrip. In the case study of the microstrip on the EBG surface, the complexity of the modal behaviors could be illustrated in the stopband. The complex mode pair in the form of $+j \alpha \pm \beta$ exists in the stopband. In the stopband of the EME microstrip, the complex mode in the form of $+j \alpha \pm \beta$ appears. Energy vanishes due to the space-wave leakages near the corner frequencies of the stopband of the microstrip on the EBG ground plane and the EME microstrip. The dispersion characteristics of three case studies exhibit the different modal behaviors although the scattering analyzes show the similar results in the passband, stopband, and radiation regions.
\end{abstract}

Index Terms-Electromagnetic bandgap (EBG), matrix-pencil method, periodical structure, photonic bandgap (PBG).

\section{INTRODUCTION}

$\mathbf{T}$ HE propagation of waves in periodical structures has been a subject of continuing interest over recent decades [1]-[6]. A survey of early research in this area reveals that various terminologies have been used according to the domain of application, such as photonic bandgap (PBG), electromagnetic bandgap (EBG), and frequency selective surfaces (FSSs) [7]-[15]. Periodical structures have attracted much interest

Manuscript received November 30, 2003; revised September 23, 2004. This work was supported by the National Science Council of Taiwan under Contracts NSC 93-2752-E-002-009-PAE and 93-2219-E-009-025.

Y.-C. Chen is with the Department of Communication Engineering, National Chiao Tung University, Hsinchu, Taiwan, R.O.C. (e-mail: henrychen@ mail.nctu.edu.tw).

C.-K. C. Tzuang is with the Graduate Institute of Communication Engineering, National Taiwan University, Taipei, Taiwan, R.O.C. (e-mail: cktzuang@cc.ee.ntu.edu.tw).

T. Itoh is with the Department of Electrical Engineering, University of California at Los Angeles, Los Angeles, CA 90095, USA (e-mail: itoh@ee.ucla.ed)u.

T. K. Sarkar is with the College of Engineering \& Computer Science, Syracuse University, Syracuse, NY 13244-1240, USA (e-mail: tksarkar@ syr.edu).

Digital Object Identifier 10.1109/TAP.2004.840536 among microwave engineers, primarily because of their potential applications in the design of waveguides and transmission line systems, which follow from their attractive slow-wave and stopband guided-wave characteristics [8]-[20]. With the advent of photonic bandgap materials in the field of optics, the development of various planar periodical transmission line structures and various EBG structures for microwave integrated circuits has attracted rapidly increasing interest. Applications including a nonleaky conductor-backed coplanar waveguide and an aperture-coupled fed patch antenna have been demonstrated exploiting deep and wide stopbands and the surface-wave suppressions (or the so-called STOP characteristic) of periodical structures [8], [9], [21]. The use of the so-called uniplanar compact photonic-bandgap (UC-PBG) structure, which is a thin planar EBG structure without via holes, opens up the possibility of integrating antennas on substrates with high dielectric constants without loss of performance. The gain and bandwidth of a microstrip antenna can be greatly enhanced using an appropriately designed artificial EBG material [11], [12]. The electric-magnetic-electric (EME) microstrip incorporates a novel EBG structure exhibiting low-loss and slow-wave bound-mode propagations. It has been utilized in a dual-frequency EME microstrip with two leaky-wave regions with radiation characteristics similar to those of the microstrip $\mathrm{EH}_{1}$ mode [16]-[19]. Compact spurious-free bandpass filters applying the inherent stopband behavior of the EBG structures to reject higher order passbands and broad-band power amplifiers using dielectric EBG structures have also been demonstrated [9], [10], [16].

Electrically investigating the periodical guiding structures embedded in many formerly unimaginable successful designs has yielded only few explications of the details of the modal behaviors of periodical structures. Although the scattering parameters have proven useful for designing the periodical structures and PBG structures, knowledge of corresponding guided-wave propagation characteristics remains very limited, especially knowledge of the physics of the stopband. This lack of insight limits the potential applications of periodical structures.

This paper raises a question regarding whether modes can exist in the stopband or not. If the answer is true, what are they like and how are such modes related to the null transmission of electromagnetic energy in the stopband? (For example, Fig. 3 of [20] shows existence of solutions with real phase constants connecting various stopbands. There is, however, no indication of 
modal solutions in the stopband.) The answers to these questions will be vital in gaining more physical insights into stopbands of periodical structures. To the authors' best knowledge, the analytical methodology utilized to explore the dispersion characteristics in the stopband has never been mentioned in the literatures. This paper provides a numerical method for investigating the modal behaviors of the periodical structures and classifying the various waves that may exist in such guiding structures. The original results answer both of the above questions, based on several case studies, including one of a microstrip subjected to even-symmetric periodical perturbations, the microstrip on the EBG surface, and the EME microstrip. Although the complex modal behaviors of the microstrip on the EBG ground plane and the EME microstrip have been described, this paper presents more precise numerical results [22]. The understanding of the modal characteristics of the EBG unit cell upon the EME microstrip has led to various applications of such EBG cells' being successfully developed by the authors' group [16]-[19]. More interesting applications based on the EBG surface are expected to be obtained by elucidating the tangled wave phenomena associated with the microstrip on the EBG structure.

Following a brief introduction, Section II reports the applications of the basic theory to analyzing periodical structures; this section introduces the numerical methodologies used herein. Section III reports the dispersion characteristics of the microstrip with periodical even-symmetric perturbations. Notches etched on the sides of the microstrip weakly perturb the periodical planar structures. The original dispersion curves of the $\mathrm{EH}_{0}$ mode (or the $\mathrm{EH}_{1}$ mode) are not substantially altered, and the interaction between various space harmonics yields further modal behaviors in the periodical structures. Section IV presents the modal behaviors of the microstrip on the EBG surface and of the EME microstrip. In the stopband of the planar periodical structures with tiny periodical lattices, the original dispersion characteristics of the fundamental space harmonic are diversified and involve extraordinary wave behaviors. Various periodical structures considered in the literature can be classified into two groups according to the modal behaviors in the stopband of the periodical structures. Section V summarizes and draws conclusions.

\section{EXTRACTIONSOF COMPLEX PROPAGATION CONSTANTS AND PRESENTATIONS OF MODAL DATA FOR PERIODICAL STRUCTURES}

Throughout this paper, the one-dimensional (1-D) periodical structure supports the propagation of waves in the longitudinal $x$ direction. The following symbols are listed for [23]:

- $e^{j \varpi t}$ : time-harmonic factor of angular frequency $\varpi=$ $2 \pi f$

- $e^{-j \gamma_{m, n} x}: \mathrm{x}$-dependence factor;

- $\gamma_{m, n}^{ \pm}=\beta_{m, n}^{ \pm}-j \alpha_{m, n}^{ \pm}: \quad \gamma_{m, n}^{ \pm}$is the complex propagation constant;

- $\beta_{m, n}^{ \pm}\left(\alpha_{m, n}^{ \pm}\right)$: propagation constant (attenuation constant) of the $m$ th higher order mode $\left(\mathrm{EH}_{m}\right)$ in association with the $n$th higher order space harmonic;

- forward traveling wave [24]: $\beta_{m, n}^{ \pm} \succ 0, \alpha_{m, n}^{ \pm}=0$;

- backward traveling wave [24]: $\beta_{m, n}^{ \pm} \prec 0, \alpha_{m, n}^{ \pm}=0$;
- forward wave [5]: $\left(\beta_{m, n}^{ \pm}\right) \cdot\left(\partial \beta_{m, n}^{ \pm} / \partial \varpi\right) \succ 0$;

- backward wave [5]: $\left(\beta_{m, n}^{ \pm}\right) \cdot\left(\partial \beta_{m, n}^{ \pm} / \partial \varpi\right) \prec 0$;

- $\gamma_{m, n}^{+}=\beta_{m, n}^{+}-j \alpha_{m, n}^{+}$: waves with group velocity $v_{g}=$ $\left(\partial \beta_{m, n}^{ \pm} / \partial \varpi\right)^{-1}>0$

- $\gamma_{m, n}^{-}=\beta_{m, n}^{-}-j \alpha_{m, n}^{-}$: waves with group velocity $v_{g}=$ $\left(\partial \beta_{m, n}^{ \pm} / \partial \varpi\right)^{-1}<0$;

Since the guiding structure is a 1-D periodical structure, Floquet's theorem mandates that the surface current $J_{s}$ on the ground plane, or on the microstrip line, are the sum of an infinite number of traveling wave components [6]. According to the feeding structure and the geometric design of the guiding structures, various modes may be excited, including the dominant $\mathrm{EH}_{0}$ mode, the first higher order $\mathrm{EH}_{1}$ mode, the second higher order $\mathrm{EH}_{2}$ mode, and others. Hence, the surface current $J_{s}$ (the surface current amplitude $J_{x}$ is applied in our case studies), simultaneously incorporating the contribution of the dominant $\mathrm{EH}_{0}$ mode and the higher order modes, as well as their fundamental and higher order space harmonics, could be generally expressed as follows [25].

$$
\begin{aligned}
J_{s} \approx & \sum_{m=-\infty}^{\infty} \sum_{n=-\infty}^{\infty} J_{m, n}^{ \pm} e^{\mp j \gamma_{m}, n} x \\
J_{s} \approx & J_{0,0}^{+} e^{-j \gamma_{0,0} x}+\sum_{n=1}^{\infty} J_{0, n}^{+} e^{-j \gamma_{0, n} x}+\sum_{n=-1}^{-\infty} J_{0, n}^{+} e^{-j \gamma_{0, n} x} \\
& +J_{0,0}^{-} e^{j \gamma_{0,0} x}+\sum_{n=1}^{\infty} J_{0, n}^{-} e^{j \gamma_{0, n} x}+\sum_{n=-1}^{-\infty} J_{0, n}^{-} e^{j \gamma_{0, n} x} \\
& +J_{1,0}^{+} e^{-j \gamma_{1,0} x}+\sum_{n=1}^{\infty} J_{1, n}^{+} e^{-j \gamma_{1, n} x}+\sum_{n=-1}^{-\infty} J_{1, n}^{+} e^{-j \gamma_{1, n} x} \\
& +J_{1,0}^{-} e^{j \gamma_{1,0} x}+\sum_{n=1}^{\infty} J_{1, n}^{-} e^{j \gamma_{1, n} x}+\sum_{n=-1}^{-\infty} J_{1, n}^{-} e^{j \gamma_{1, n} x}+\cdots \\
& +J_{m, 0}^{+} e^{-j \gamma_{m}, 0}+\sum_{n=1}^{\infty} J_{m, n}^{+} e^{-j \gamma_{m, n} x} \\
& +\sum_{n=-1}^{-\infty} J_{m, n}^{+} e^{-j \gamma_{m, n} x} \\
& +J_{m, 0}^{-} e^{j \gamma_{m, 0} x}+\sum_{n=1}^{\infty} J_{m, n}^{-} e^{j \gamma_{m, n} x} \\
& +\sum_{n=-1}^{-\infty} J_{m, n}^{-} e^{j \gamma_{m, n} x}
\end{aligned}
$$

where

$$
\gamma_{m, n}^{ \pm}=\beta_{m, n}^{ \pm}-j \alpha_{m, n}^{ \pm}=\left(\beta_{m, 0}^{ \pm} \pm \frac{2 \pi}{d} n\right)-j \alpha_{m, 0}^{ \pm} .
$$

In (2), $J_{0, n}^{+}$and $J_{m, n}^{+}$components represent the current amplitudes of the dominant $\left(\mathrm{EH}_{0}\right)$ and $m$ th higher order $\left(\mathrm{EH}_{m}\right)$ forward-traveling waves (both with positive phase velocity) associated with the $n$th higher order space harmonic, respectively. On the other hand, the current amplitudes with a minus sign in their superscripts $\left(J_{m, n}^{-}\right)$represent the fundamental and $n$th higher order space harmonics subject to the dominant or higher order backward-traveling waves [25]. 


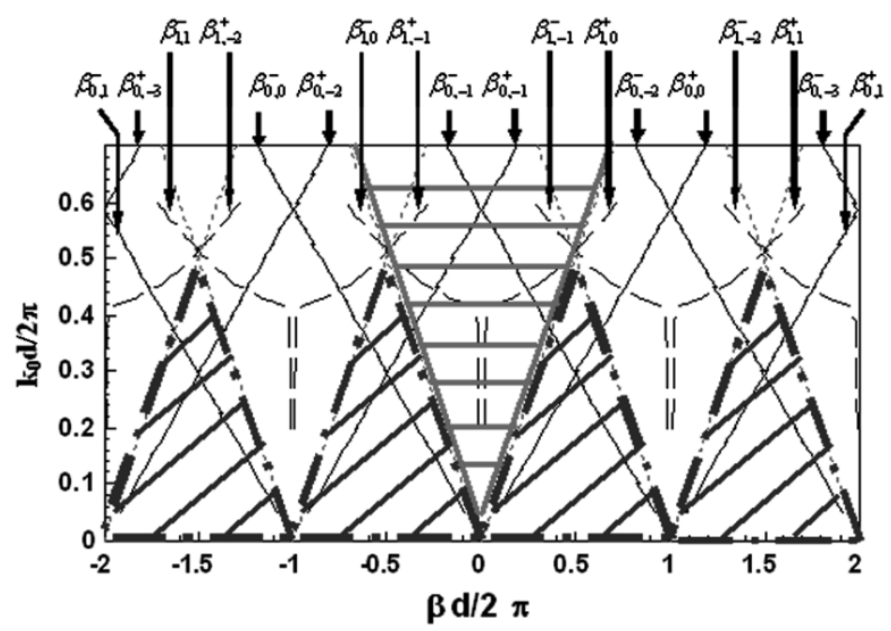

Fig. 1. Template of Brillouin diagrams for the perturbed microstrip structures with periodical loadings. The lined region and the oblique-lined triangle regions represent the radiation region and the completely bound regions, respectively.

Fig. 1 shows the template of the Brillouin diagram, or the so-called $k-\beta$ diagram, of the periodical structure [25], [26]. The dispersion characteristics of the microstrip structure with a width equal to that in the case study considered in Section III are applied here to generate the template for the Brillouin diagram. The solid lines labeled $\beta_{0, n}^{ \pm}$and the dashed lines labeled $\beta_{1, n}^{ \pm}$represent the dominant $\mathrm{EH}_{0}$ mode and the first higher order $\mathrm{EH}_{1}$ mode in association with the $n$th higher order space harmonic, respectively. Notably, the normalized phase constant of the original dominant $\mathrm{EH}_{0}$ mode $\left(\beta_{0,0}^{+}\right)$in the case study considered here is about 1.8 and the onset frequency of the original first higher order $\mathrm{EH}_{1}$ mode is $10.0 \mathrm{GHz}$. The periodicity of the perturbations applied to the uniform microstrip is specifically selected to be the half-wavelength of the free-space TEM wave at the onset frequency of the $\mathrm{EH}_{1}$ mode.

(1) and (2) assume that the current distributions along the microstrip line are exponential waveforms with respect to the longitudinal axis, and that the matrix-pencil method can be applied to analyze the surface current in the periodical structures [25]-[27]. Therefore, the following expression is a valid approximation to the surface current, $J_{s}$ :

$$
J_{s}(x, y) \approx \sum_{i=1}^{M} J_{i}(y) e^{-j \gamma_{i} x} \quad \mathrm{M}=1,2,3, \ldots
$$

where $J_{s}(x, y)$ is a known quantity obtained by solving the periodical structure by invoking the method of moments. In this paper, the surface current distribution $J_{s}(x, y)$ of the periodical structure - either on the ground plane or on the above signal line - is obtained by launching the dominant bound $\mathrm{EH}_{0}$ mode at one end of the strip line of the periodical structure with the other end under matched termination. $\gamma_{i}$ is the extracted complex propagation constant of one wave component obtained without prior knowledge of the dominant mode $\left(\mathrm{EH}_{0}\right)$ or higher order modes $\left(\mathrm{EH}_{1}, \mathrm{EH}_{2}\right.$, or $\left.\mathrm{EH}_{m}\right)$ or their space harmonics. The integer $M$ in (4) represents the number of the types of traveling waves applied to approximate the current distributions on the periodical structure. $J_{i}(y)$ is the extracted current amplitude of the $i$ th traveling wave component at location $y$ in the transverse plane. Notably, the criterion by which the mode number $M$ is chosen involves the relative current amplitudes of the wave components. The ratio $\sigma_{M} / \sigma_{M+1}$, which is the ratio of the square of the relative current amplitude of the two wave components $J_{M}(y) e^{-j r_{M} x}$ and $J_{M+1}(y) e^{-j r_{M+1} x}$, can be used to decide the value of $M$ required to obtain the assumed precision [27].

Next, the finite-element HFSS $^{1}$ is applied to verify the theoretical results obtained by the matrix-pencil technique. The prototype periodical microstrip, used in simulation by the finite-element method, has one period in the longitudinal direction with the linked boundary condition (LBC) at the cell sidewalls to simulate periodicity. An open boundary normal to the direction of propagation can be modeled accurately using perfectly matched layers (PML). The guided wavenumbers predicted using a typical full-wave integral equation solver and Floquet's theorem are applied here as the initial guess of the finite-element simulator. The following sections will show excellent agreements between the results obtained by the matrix-pencil and the finite-element methods in the passband and the radiation region in the case studies. However, the wave components in the stopband are hard to derive in the finite-element simulation.

\section{ONE-Dimensional TRANSMisSion LiNES With Half-Wavelength Periodical Perturbations: A WEAKLY AgITATED TYPE}

We propose a simplified model as shown Fig. 2 for studying the dispersion characteristics of a microstrip subject to the periodical perturbations, which are classified as weakly agitated type when compared with those studied in Section IV. The inductive loads made of notches, in even-symmetric $\left(d_{\text {offset }}=\right.$ 0 ) fashions, create perturbations on the unperturbed microstrip $\mathrm{EH}_{0}$ bound mode launched at the input end. We invoke method of moment solver IE3 $\mathrm{D}^{2}$ for obtaining the surface currents $J_{s}$, which in this particular case study, flows in the bottom, uniform ground plane underneath the microstrip. Extensive numerical analyzes employing the method of moments (MoM) and the matrix-pencil method show that the propagation constants of the space harmonics converge to within $1 \%$ when the total length of the transmission line is greater than nine periods. Such numerical accuracy for the particular periodical structures of interest mandates attentions for the following aspects. In the method of moment simulation, the grid size in the central area of both top metal strip and bottom ground plane is kept smaller than 12 cells per unit wavelength and additional grids with width of only $0.02 \mathrm{~mm}$ should be added alongside the edges of the top metal strip for improving accuracy. A series of numerical experiments confirms that the above-mentioned meshing scheme is sufficient for the converged solutions in the MoM analyzes. Next, extensive matrix-pencil analyzes of the same guiding structure further show that more than 11 equally sampled points per wavelength of $\beta_{0,0}^{+}$component at the highest operating frequency in the $x$ direction ( $1.57 \mathrm{~mm}$ in the particular case study) and 7 equal sampled locations in the $y$ direction $(1.43 \mathrm{~mm}$ in the particular case

${ }^{1}$ HFSS is a registered trademark of Ansoft Corporation.

${ }^{2}$ IE3D is a registered trademark of Zeland Software Inc. 


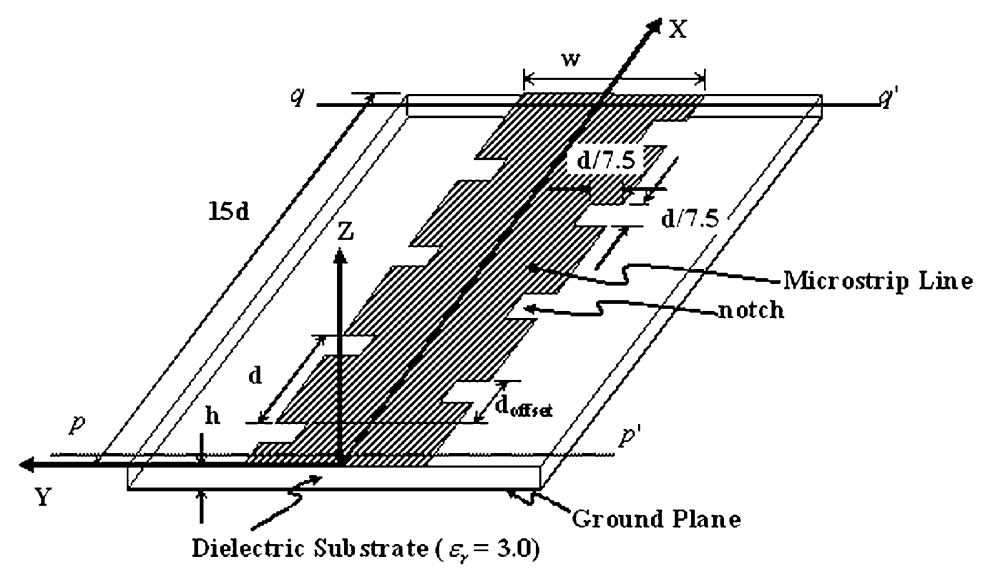

Fig. 2. Microstrip models with periodical perturbations; structure parameters and material constants: $w=10 \mathrm{~mm}, h=0.254 \mathrm{~mm}, d=15 \mathrm{~mm}, \varepsilon_{r}=$ $3.0, d_{\text {offset }}=0$. Substrate is RO3003 ${ }^{\mathrm{TM}}$.

study) will result in accurate estimates of the space harmonics extracted from $J_{s}$ obtained previously by the MoM method.

Fig. 3 shows the theoretical two-port scattering parameters, between the reference planes $p-p^{\prime}$ and $q-q^{\prime}$ of Fig. 2, showing a stopband with input reflection coefficient $\left(S_{11}\right)$ approaching $0 \mathrm{~dB}$ between 5.2 and $6.2 \mathrm{GHz}$ approximately. Below the stopband, the bound $\mathrm{EH}_{0}$ mode, which will be specifically designated as the $\beta_{0,0}^{+}$forward traveling wave in this section later, survives the periodical perturbations and reaches port 2 without encountering much losses. The input transmission coefficients $\left(S_{21}\right)$ show a sudden decrease in magnitude with $20 \mathrm{~dB}$ attenuation and the input reflection coefficient near $0 \mathrm{~dB}$ in the stopband. Therefore the electromagnetic power can hardly propagate in such guiding structure with a stopband. What plotted on the right-hand axis of Fig. 3 are the values of RPA (relative power absorbed, $1-\left|S_{11}\right|^{2}-\left|S_{21}\right|^{2}$ ) [18]. The relative power absorbed (RPA) represents the total power losses of the electromagnetic device under investigation. Pictorially Fig. 3 shows three zones of interests, namely:, 1) propagating region below the stopband; 2) stopband; and 3) propagating region above the stopband with considerable losses.

Applying the matrix-pencil method as mentioned in Section II, we then extract various traveling wave components from the surface current $J_{s}$ along the longitudinal $x$ direction at $y=0$ underneath the periodical microstrip. The results are plotted in the form of Brillouin diagram as shown in Fig. 4(a). The normalized phase constants of the traveling wave components extracted by the matrix-pencil method are represented in circle symbols and are in excellent agreement with the prediction based on the Floquet's theorem. Next the finite-element method provides another supplemental checks by confirming the theoretical results obtained by the matrix-pencil method. The solutions obtained by the finite-element method are marked in the empty triangles, which agree excellently with the results obtained by the matrix-pencil method outside the stopband. $\beta_{0,0}^{+}$component represents the forward traveling wave of the fundamental microstrip mode $\left(\mathrm{EH}_{0}\right.$ mode), which in the particular case study has nearly the same phase constant as that of $\beta_{0,0}^{+}$. Fig. 4(a) shows that $\beta_{0, n}^{ \pm} d / 2 \pi-\beta_{0,0}^{ \pm} d / 2 \pi$ is indeed $\pm n$ in the horizontal axis, confirming that $\beta_{0, n}^{ \pm}$is the space harmonic of $\beta_{0,0}^{ \pm}$component of order $n$ predicated by (3). Near $5.1 \mathrm{GHz}$

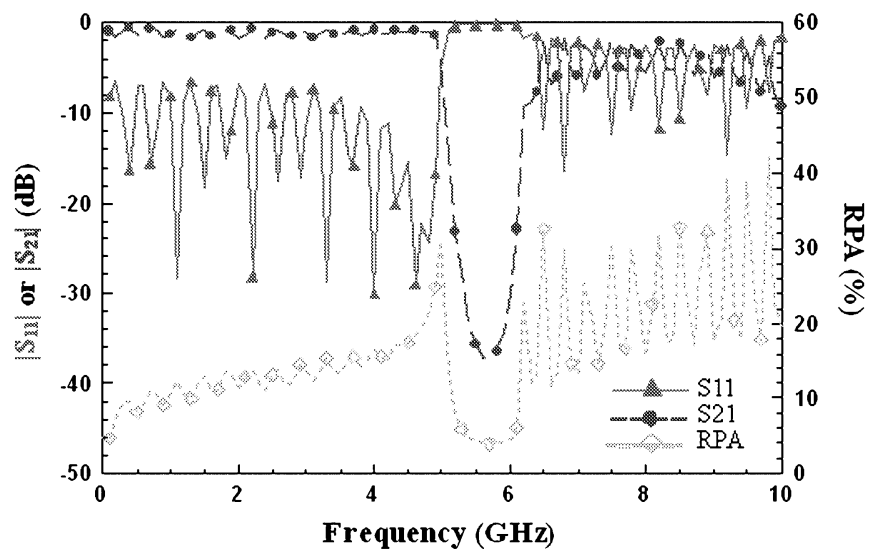

Fig. 3. Theoretical two-port scattering parameters (left axis), and RPA values (right axis) of the microstrip line with periodical perturbations of even-symmetries.

$(\beta d / 2 \pi=0.5), \beta_{0,0}^{+}$and $\beta_{0,-1}^{-}$dispersion curves emerge in the frame (a1+) of Fig. 4(a), so do the modes $\beta_{0,1}^{+}$and $\beta_{0,-2}^{-}$at $\beta d / 2 \pi=1.5$ in the frame $(\mathrm{a} 2+)$, modes $\beta_{0,-1}^{+}$and $\beta_{0,0}^{-}\left(\beta_{0,-2}^{+}\right.$ and $\left.\beta_{0,1}^{-}\right)$in the frame $(\mathrm{a} 1-)$ [the frame $(\mathrm{a} 2-)$ ]. Between 5.1 and $6.1 \mathrm{GHz}$, there exist stopbands periodically located at $\beta d / 2 \pi=-1.5,-0.5,0.5$, and 1.5 , respectively. The Brillouin diagram explicitly determines the position of the stopband without ambiguity, which occurs in the scattering analyzes with uncertainty about the corner frequencies of the stopband.

Next we focus on the dispersion curves bounded by $\beta d / 2 \pi=-1$ and $\beta d / 2 \pi=1$. Fig. 4(b) shows the expanded views of the Brillouin diagram surrounding the stopband [see the frames $(\mathrm{a} 1+)$ and (a1-) of Fig. 4(a)]. Circular and triangle symbols represent the normalized phase constants $\beta d / 2 \pi$ and the normalized attenuation constants $\alpha d / 2 \pi$, respectively. In the stopband, we clearly observe the formation of complex modes, which show the normalized phase constants of nearly \pm 0.5 symmetric about the Brillouin diagram. The normalized attenuation constants of the complex modes, on the other hand, form an ellipse in the stopband. This is also in good agreement with the prediction based on (18) of [23], which explains the formation of complex modes as the direct result of mode coupling of a forward wave $\beta_{0,0}^{+}$and a backward wave $\beta_{0,-1}^{-}$. 


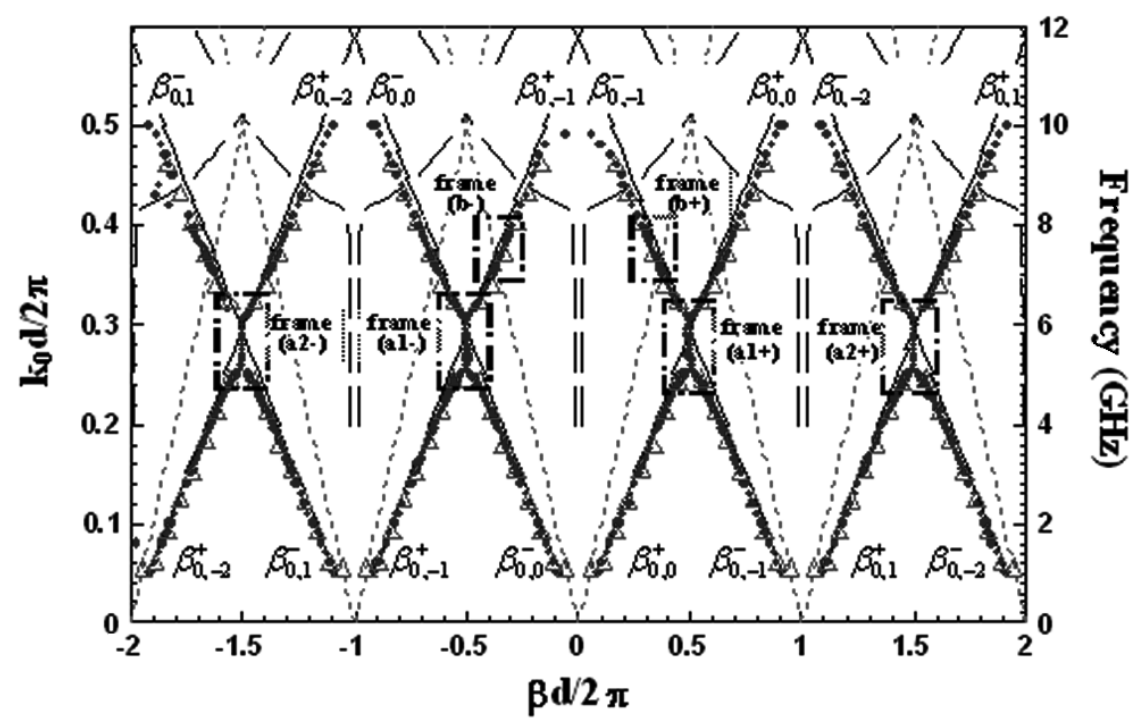

(a)

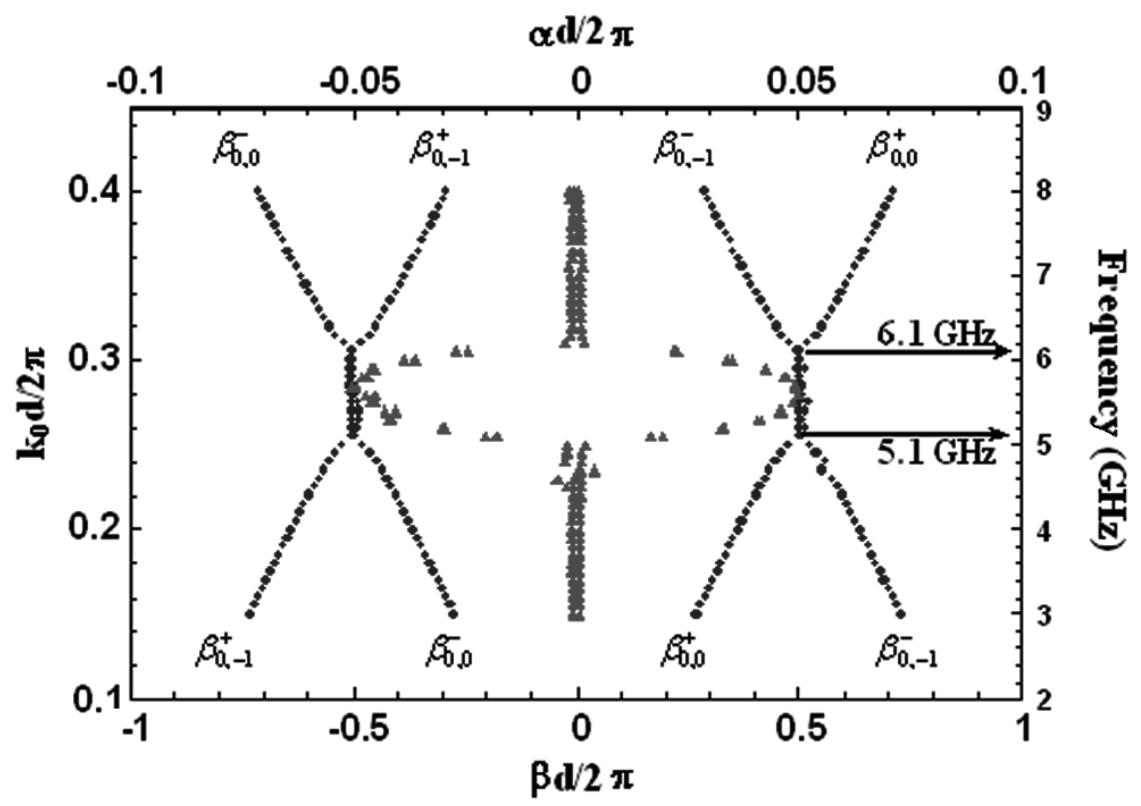

(b)

Fig. 4. Brillouin diagram of the periodic planar guiding structure of Fig. 2. The circles and the empty triangles in (a) represent the normalized phase constants obtained by invoking the matrix-pencil method and finite-element method, respectively. The circles and triangles in (b) represent $\beta d / 2 \pi$ and $\alpha d / 2 \pi$ obtained by the matrix-pencil method, respectively. (a) Brillouin diagram. (b) Details of the dispersion curves between $\beta d / 2 \pi=-1$ and $\beta d / 2 \pi=1$ in the stopband.

Similarly, $\beta_{0,-1}^{+}$and $\beta_{0,0}^{-}$are another pair of forward wave and backward wave that also couple and consequently establish a pair of complex modes. The discovery of complex modes in the periodically loaded microstrip structure is a revelation of the phenomena that complex modes can exist in an open waveguide structure in contrast to boxed or partially open structures [28]-[30].

In the stopband, the $\beta_{0,0}^{+}$and $\beta_{0,-1}^{-}\left(\beta_{0,-1}^{+}\right.$and $\left.\beta_{0,0}^{-}\right)$dispersion curves emerge on the right-half (left-half) plane. The relative current amplitudes of these two modes $\left(\beta_{0,0}^{+}\right.$and $\beta_{0,-1}^{-}$ or $\beta_{0,-1}^{+}$and $\left.\beta_{0,0}^{-}\right)$with the same phase constants $\left(\beta_{0,0}^{+} d / 2 \pi=\right.$ $\beta_{0,-1}^{-} d / 2 \pi=0.5$ or $\left.\beta_{0,-1}^{+} d / 2 \pi=\beta_{0,0}^{-} d / 2 \pi=-0.5\right)$, however, differ by two orders of magnitude. Table I shows the extracted modal current amplitudes and the corresponding nor- malized complex propagation constants at $5.8 \mathrm{GHz}$ based on the matrix-pencil method. The normalized complex propagation constants of the reported space harmonics are numbered by the descending order of current amplitudes. The first two space harmonics have relative current amplitudes much larger than the other two space harmonics. Furthermore, the first two space harmonics establish a pair of complex modes in the form of $+j \alpha \pm \beta$. The third and forth space harmonics also make the second pair of complex modes in the form of $-j \alpha \pm \beta$. Such classification of complex modes is one-to-one corresponding to those of boxed, lossless finline structures in the evanescent mode region. Omar et al. [28] reported that a pair of complex modes can carry only pure reactive power so that they behave as a whole evanescently. The obtained 
TABLE I

Complex Conjugate Propagation Constants and the Normalized CurRent Amplitude OF THE Microstrip With EVEN-SYMMETRIC PERTURBATIONS AT $5.8 \mathrm{GHz}$

\begin{tabular}{c|c|c|c}
\hline $\begin{array}{c}\text { Relative current } \\
\text { amplitude }\end{array}$ & $\begin{array}{c}\text { Normalized } \\
\text { attenuation constant }\end{array}$ & $\begin{array}{c}\text { Normalized phase } \\
\text { constant }\end{array}$ & $\begin{array}{c}\text { Complex } \\
\text { Modes }\end{array}$ \\
\hline 1.000000 & 0.1628313 & 1.723700 & $+j \alpha \pm \beta$ \\
0.997452 & 0.1607144 & -1.722872 & +1.721198 \\
\hline 0.018785 & -0.1650002 & -1.748339 & $-j \alpha \pm \beta$ \\
0.000478 & -0.1619412 & 0 \\
\hline
\end{tabular}

solution of a pair of complex modes in the stopband of a periodically perturbed transmission line is a manifestation of Omar's theory applied to an open guiding structure.

In frame $(\mathrm{a} 2+)$ [frame $(\mathrm{a} 2-)$ ] of Fig. 4(a), the dispersion curves of the wave components $\beta_{0,1}^{+}$and $\beta_{0,-2}^{-}\left(\beta_{0,-2}^{+}\right.$and $\left.\beta_{0,1}^{-}\right)$ also cross at the position $\beta d / 2 \pi=1.5(\beta d / 2 \pi=-1.5)$ and these two space harmonics $\beta_{0,1}^{+}$and $\beta_{0,-2}^{-}\left(\beta_{0,-2}^{+}\right.$and $\left.\beta_{0,1}^{-}\right)$ couple. However, only one pair of complex waves in the form of $+j \alpha \pm \beta$ could be obtained by applying the matrix-pencil analysis with the mode choice criterion. The attenuation constants of these two pair of complex modes $\left(\beta_{0,1}^{+}\right.$with $\beta_{0,-2}^{-}$and $\beta_{0,-2}^{+}$ with $\beta_{0,1}^{-}$) are nearly the same as those of the complex mode pairs located at $\beta d / 2 \pi=0.5$ and $\beta d / 2 \pi=-0.5$, agreeing with the relation of (3), and also forming an ellipse with only the right half.

When the operating frequency exceeds $7.2 \mathrm{GHz}\left(k_{0} d / 2 \pi \approx\right.$ $0.36)$, both $\beta_{0,-1}^{+}$and $\beta_{0,-1}^{-}$components enter the radiation region and become leaky modes by passing the air-TEM triangle boundaries as shown in frame $(\mathrm{b}+)$ and frame $(\mathrm{b}-)$ in Fig. 4(a). The simulated radiation efficiency, defined as the total radiation power divided by the net input power of the periodical microstrip, increase to about $15 \%$ suddenly as the operating frequency greater than about $7.3 \mathrm{GHz}$. The current amplitude of the $\beta_{0,-1}^{+}$component is about twice than that of the $\beta_{0,-1}^{-}$ component. Extensive numerical analyzes show that accurate far field radiation pattern must take into account all the major space harmonics that could radiate. At $9.0 \mathrm{GHz}$, the normalized phase constants of the $\beta_{0,-1}^{+}$and $\beta_{0,-1}^{-}$components are \pm 0.43 . Applying the relation of the normalized phase constant $\beta / k_{0}$ and the radiation angle $\theta\left(\theta=\sin ^{-1}\left(\beta / k_{0}\right)\right)$, we could find that the radiation angles from the broadside direction of these two components are $\pm 25.5^{0}$ [31]. The simulated main beam angle is approximately $25^{\circ}$ from the broadside direction in both forward and backward directions, agreeing with the prediction of the Brillouin diagram.

Limited to the space available in this paper, the dispersion characteristics of the microstrip with periodical skew-symmetric perturbations $\left(d_{\text {offset }}=d / 2\right.$ in Fig. 2) are left to an expanded report in the near future. More diversified modal behaviors, including the disappearance of the first stopband, the leaky wave leakage, and alternation of symmetry of current distributions of various space harmonics, etc., could be observed in the skew-symmetric case study.

\section{LOAded Microstrip With Periodicity Much SMALleR THAN HALF GUIDED-WAVELENGTH}

\section{A. Microstrip on the EBG Surface}

Fig. 5 shows the schematic of the popularly known EBG structure (the so-called UC-PBG structure) under investigation,

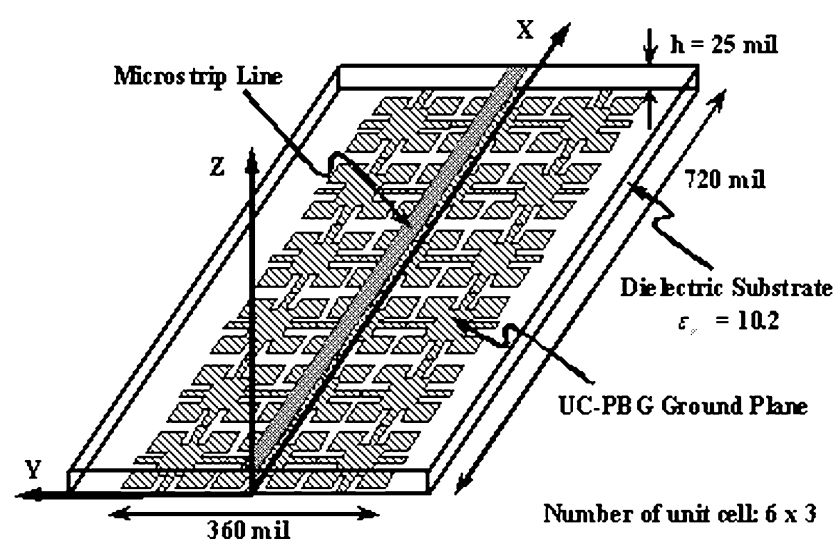

Fig. 5. Schematics of microstrip line on the EBG surface. The structural and material constants are identical to those in [9].

consisting of a microstrip line on a ground plane made by a $3 \times 6$ lattice using EBG cells. The detailed dimensions of the unit cell (or lattice) and the simulation scattering parameters were reported in [9].

Applying the matrix-pencil method, we can extract various components of waves in the guiding structure from the surface current distribution $J_{s}$ on the microstrip above the EBG surface. Cares must be exercised during method of moment simulation, notably, 12 cells per unit free-space wavelength of the highest frequency of interest and the edge grids of $0.02 \mathrm{~mm}$ on the periphery of the EBG cell are adopted for converged solutions. Upon completion of full-wave solver, matrix-pencil method steps in, analyzing the surface currents $J_{s}$ of microstrip at $z=h$, where typically 47 equally spaced sampling points per wavelength of $\beta_{0,0}^{+}$component at $20 \mathrm{GHz}$ in the longitudinal direction ( $0.10 \mathrm{~mm}$ in the particular case study) and seven equally sampled points in the transverse $y$ direction $(0.09 \mathrm{~mm}$ in the particular case study) are found to produce accurate estimates of the extracted space harmonics. Extensive numerical experiments confirm that the above-mentioned meshing scheme for the integral equation solver and matrix-pencil method is adequately sufficient for the converged space harmonic solutions of the particular periodical structure. The results extracted from the surface currents along the $x$ direction at $y=0$ are compiled in the form of Brillouin diagram, as shown in Fig. 6. Notice that the solutions obtained by the finite-element method are marked in the empty triangles, which agree excellently with the results obtained by the matrix-pencil method in the passbands. The radiation region is bounded by two free-space TEM lines. (See Fig. 1 for detail.) 

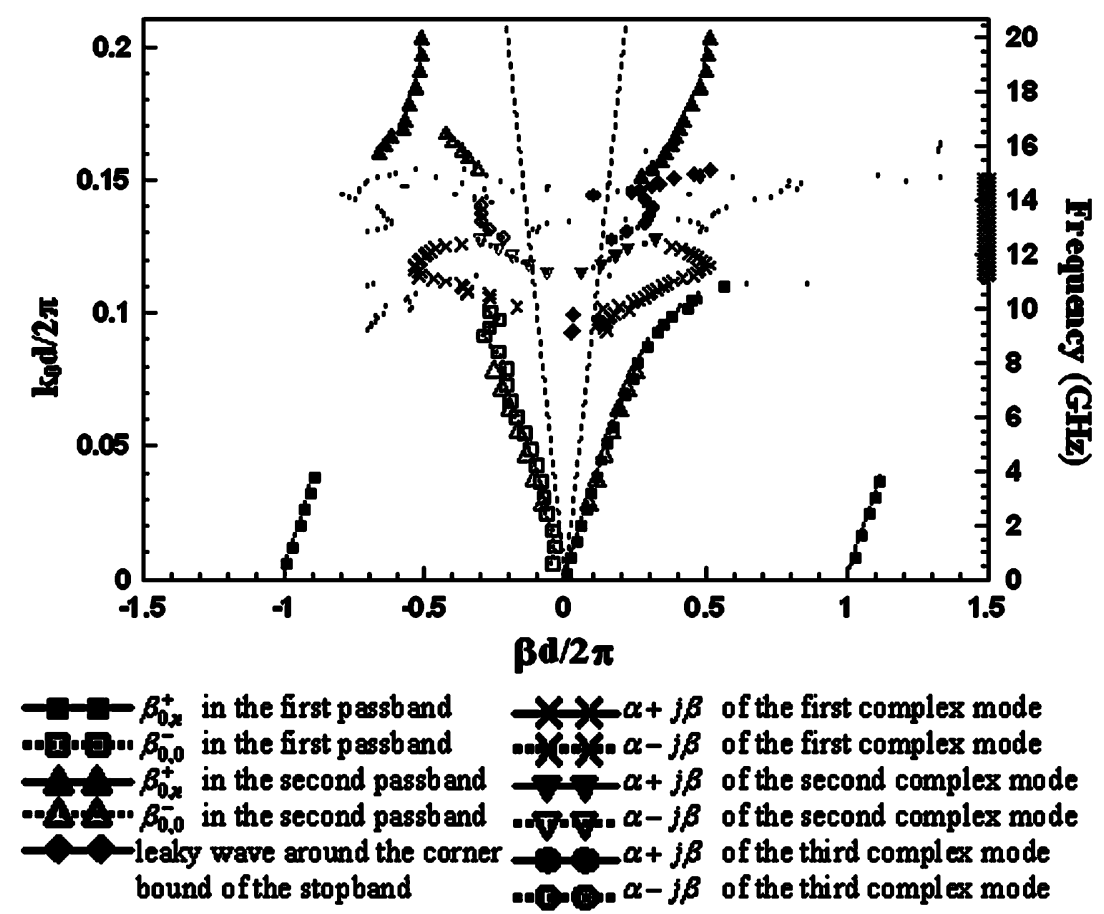

Fig. 6. Brillouin diagram of the microstrip on the EBG surface. The solid symbols and the empty triangles represent the normalized phase constants obtained by invoking the matrix-pencil method and finite-element simulation, respectively.

The modal solutions as depicted in Fig. 1 are surprisingly complicated. First, the direct bandgap-type stopband, borrowing the jargon from solid-state physics, disappears and looks like an indirect bandgap behavior. Second, the simple ellipse-shape stopband characteristics in the case of weakly perturbed microstrip structures no longer hold for the present case study. Instead we observe many complex modes in the stopband.

Investigating Fig. 6 from $1 \mathrm{GHz}$ and above, we observe that four wave components exist, namely, $\beta_{0,0}^{+}, \beta_{0,0}^{-}, \beta_{0,-1}^{+}$, and $\beta_{0,1}^{+}$, which are arranged by the descending order of relative current amplitude. In the particular case study, $\beta_{0,0}^{+}$is one order of relative current amplitude larger than $\beta_{0,0}^{-}$, which is also approximately one order of relative current amplitude larger than $\beta_{0,1}^{+}$and $\beta_{0,-1}^{+}$. Beside the FEM checks, the obtained space harmonics also validate the theoretical approach adopted in this paper. The $\beta_{0,0}^{+}, \beta_{0,-1}^{+}$, and $\beta_{0,1}^{+}$components show similar modal current distribution to the conventional microstrip mode. Based on the criterion that $\sigma_{M} / \sigma_{M+1}>8$, means that the excited $(M+1)$ th component carry negligible power when compared with the previous lower order component. Only $\beta_{0,0}^{+}$ and $\beta_{0,0}^{-}$components prevail for frequency above $4 \mathrm{GHz}$ based on the mode selection criterion.

The dispersion curves of $\beta_{0,0}^{+}$and $\beta_{0,0}^{-}$should show a reflection symmetry about the vertical axis at $\beta d / 2 \pi=0$. Fig. 6 , however, shows that $\beta_{0,0}^{-}$is not quite symmetrical against $\beta_{0,0}^{+}$ since the amplitude of $\beta_{0,0}^{-}$component is about $1 / 20$ of $\beta_{0,0}^{+}$ and numerical inaccuracy occurs during the matrix-pencil extraction process. The dispersion curve of the $\beta_{0,0}^{+}$mode, on the other hand, should be symmetrical about the $\beta d / 2 \pi=0.5$ axis. The disappearing half of the $\beta_{0,0}^{+}$mode is, in principle, the $\beta_{0,-1}^{-}$ component, which is the space harmonic of $\beta_{0,0}^{-}$that is already weakly excited. Therefore this $\beta_{0,-1}^{-}$component does not appear in the Brillouin diagram. Of more importance is the fact that the group velocity of $\beta_{0,0}^{+}$at $\beta d / 2 \pi=0.5$, also corresponding to $10.6 \mathrm{GHz}$, is zero. For the first time we may rigorously determine the lower bound stopband frequency of the complex periodical structure that merges a microstrip line and a two-dimensional (2-D) periodical structure. The upper bound of the stopband is approximately at $14.9 \mathrm{GHz}$, where the group velocity of the $\beta_{0,0}^{+}$mode is approaching zero.

Between 10.6 and $14.9 \mathrm{GHz}$, we observe birth and death of complex modes, interleaving in the stopband. Fig. 7 illustrates the interleaving phenomena alternating of the complex modes by plotting normalized phase constant $\left(\beta / k_{0}\right)$, relative current amplitude, and normalized attenuation constant $\left(\alpha / k_{0}\right)$, respectively, in parts (a)-(c) of the figure. Notice that the modal current ratio of $\beta_{0,0}^{+}$over $\beta_{0,0}^{-}$is greater than 10 below $7 \mathrm{GHz}$ [see Fig. 7(b)]. In the lower passband, the slow-wave factor $\left(\beta / k_{0}\right)$ of the $\beta_{0,0}^{+}$forward traveling wave component is approximately 1.07-to- 1.62 than that of an ordinary $50-\Omega$ line of the same width on a uniform ground plane.

As the operating frequency reaches $8 \mathrm{GHz}, \beta_{0,0}^{+}$becomes weaker, and its modal amplitude decrease abruptly to zero at 10.6 GHz, the lower bound of the stopband (see Fig. 7(b) for the solid line with solid square symbols). When the $\beta_{0,0}^{+}$mode approaches the stopband, the normalized phase constant (or the SWF) increases indefinitely to infinity [see Fig. 7(a)] and the normalized attenuation shows an abrupt increase [see Fig. 7(c)]. Observing closely between 9.1 and $9.8 \mathrm{GHz}$, predominantly space-wave leakage emerges as illustrated by the solid lines with diamond symbols in Fig. 7. The relative current amplitude of this leakage can be much higher than the $\beta_{0,0}^{+}$component, 


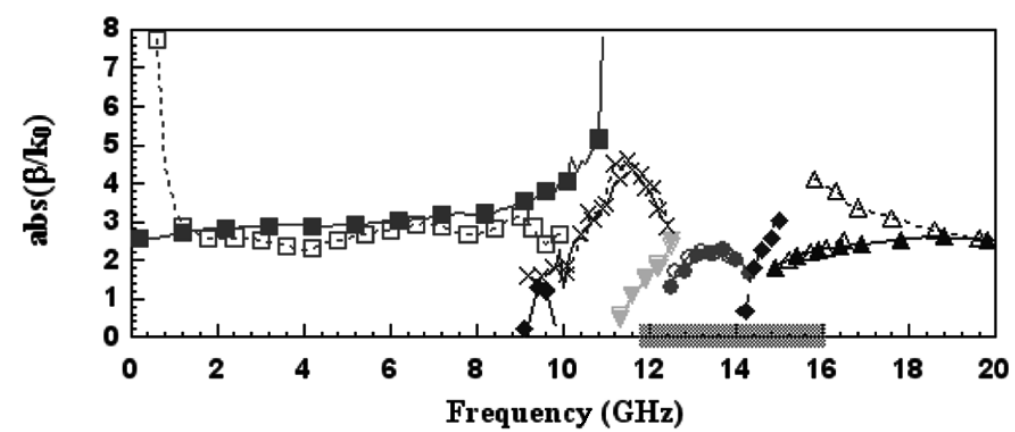

(a)

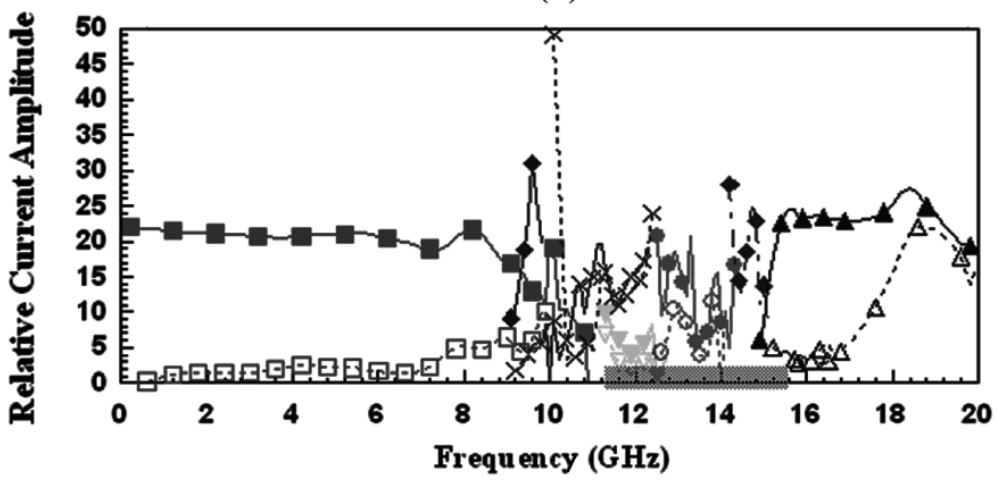

(b)

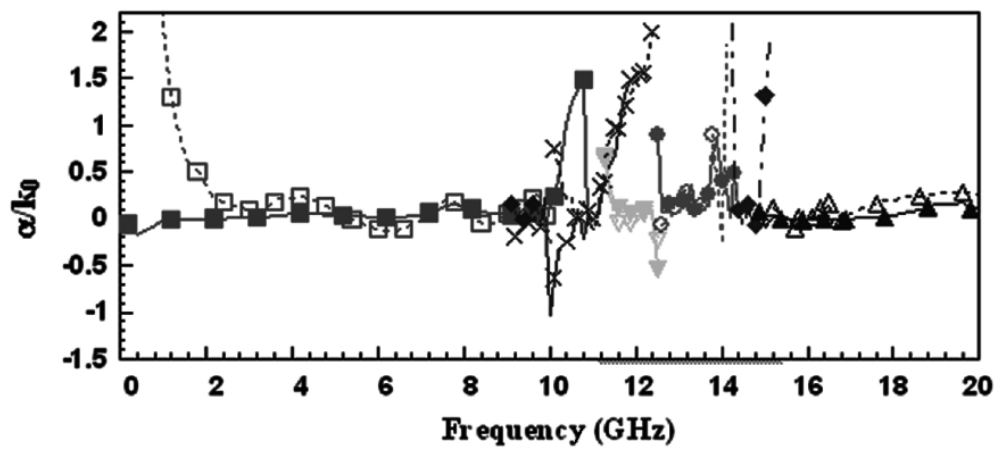

(c)

Fig. 7. Complex propagation constants and the relative current amplitudes of the EBG structure in the various regions of interest. (a) Normalized phase constants, (b) relative current amplitudes, and (c) normalized attenuation constants.

explaining the potential hazards of using the EBG cells as surface-wave suppressors as reported in [32]. Between 9.1 and $9.8 \mathrm{GHz}$, the EBG ground plane actually enhances the coupling between two microstrip open stubs as seen in Fig. 5(b) of [8] and Fig. 6 of [32].

Fig. 6 shows that three pairs of complex modes appear in the stopband alternately. The first pair, in cross symbols, in the form of $+j \alpha \pm \beta$, starts at $9.2 \mathrm{GHz}$ below $10.6 \mathrm{GHz}$ (the lower bound for the stopband). Notice that two sets of $\beta^{\prime} s$ are overlaid in Fig. 7(a), since the absolute values are displaced. The magnitudes of the phase constants of the complex mode are nearly equal for all three pairs in the stopband. The corresponding attenuation constants of the complex modes for these three cases are almost the same. The second pair of the complex modes emerges before the disappearance of the first pair; the second pair begins with $11.3 \mathrm{GHz}$ and ends at $12.5 \mathrm{GHz}$. The third pair starts at $12.5 \mathrm{GHz}$ and ends at $14.4 \mathrm{GHz}$, respectively. Notice that the amplitudes of the complex modes of each pair should be equal theoretically. We notice that the first pair has nearly equal amplitude, whereas the third pair has slight difference in magnitude. It is also interesting of observe that second leaky wave emerges in the stopband and before the second passband appears. Between 14.2 and $15.1 \mathrm{GHz}$, this leaky wave, which is initially a space wave leakage in the forward direction at $14.2 \mathrm{GHz}$, becomes a surface wave at $14.3 \mathrm{GHz}$ and beyond. Appreciable amount of this leaky wave is excited in the particular case study, since its relative current amplitude is larger than those of the complex modes.

Above $15.2 \mathrm{GHz}$, the forward traveling wave $\beta_{0,0}^{+}$(the solid line with solid triangles) returns since it has similar modal current distributions as those of $\beta_{0,0}^{+}$below $10.6 \mathrm{GHz}$ and the backward traveling wave $\beta_{0,0}^{-}$(the dash line with empty triangles) also emerges. Notice that Fig. 7(b) shows 
that the relative current amplitudes of the $\beta_{0,0}^{+}$wave in the first and second passbands are nearly equal. Also notice that two passbands are observed for the $\beta_{0,0}^{+}$mode. The space harmonic $\mathrm{n}=-1$ of the $\beta_{0,0}^{+}$in the second passband also emerges.

\section{B. EME Microstrip}

The EME microstrip is implemented by adding the EBG cells alongside the metal strip(s) in the axial direction [16]-[19]. The geometry, dimensions, and material constants of the EBG cell, latticed on the metal strip line of the EME microstrip are shown in Fig. 8, where part (a) shows an EME microstrip of single cell in the transverse direction and part (b) illustrates the detail of the cell.

The same extraction procedures for obtaining the Brillouin diagram of the EME microstrip are applied. Here $J_{s}$ denotes the ground returned currents underneath on the bottom ground plane. 12 cells per unit wavelength and the additional edge grids of width $0.02 \mathrm{~mm}$ alongside the unit cells are applied. On the ground plane of the EME microstrip, we adopt more than ten equally sampling points per wavelength of $\beta_{0,0}^{+}$component at $15 \mathrm{GHz}$ in the longitudinal direction $(1.09 \mathrm{~mm}$ in the particular case study) and night equally sampled grids in the transverse direction (1.13 $\mathrm{mm}$ in the particular case study) for extract accurate estimates of the space harmonics extracted from the surface currents $J_{s}$. The results extracted from the surface currents along the $x$ direction at $y=0$ are compiled to form the Brillouin diagram as shown in Fig. 9. The solutions obtained by the finite-element method are marked in the empty triangles, which agree excellently with the results obtained by the matrix-pencil method in the first passband below the stopband. To the left-hand side of Fig. 9 are the plots of the normalized attenuation constants $\left(\alpha / k_{0}\right)$ and the relative current amplitudes of the various wave components. Only two dominate space harmonics, namely, the $\beta_{0,0}^{+}$and $\beta_{0,0}^{-}$modes are shown in Fig. 9; the rest are filtered out by the mode selection criterion.

In the low frequency region, $\beta_{0,0}^{+}$and $\beta_{0,0}^{-}$are respectively the forward and the backward traveling waves with negligibly small attenuation constants. The group velocity of the $\beta_{0,0}^{+}$ (also $\beta_{0,0}^{-}$) mode approaches zero near $8.6 \mathrm{GHz}$, which is defined as the lower bound frequency of the stopband. Similar to the previous case study (Section IV-A), an additional leaky wave exists before the stopband occurs. Between 7.5 and $8.5 \mathrm{GHz}$, a leaky wave (the solid line with diamonds) in the forward direction emerges. Such finding can be correlated to the significant increase of the RPA value shown in Fig. 10, where the RPA value of $85 \%$ is peaked at $8.3 \mathrm{GHz}$, reflecting significant amount of losses in electromagnetic energies. When observing carefully Fig. 10, the upper corner frequency of the stopband is near $9.4 \mathrm{GHz}$, at which the group velocity of the complex modes approaches zero. Therefore, we may clearly define the stopband is indeed between 8.5 and $9.4 \mathrm{GHz}$. In the stopband, both normalized attenuation constant and relative current amplitude of the complex modes are equal,

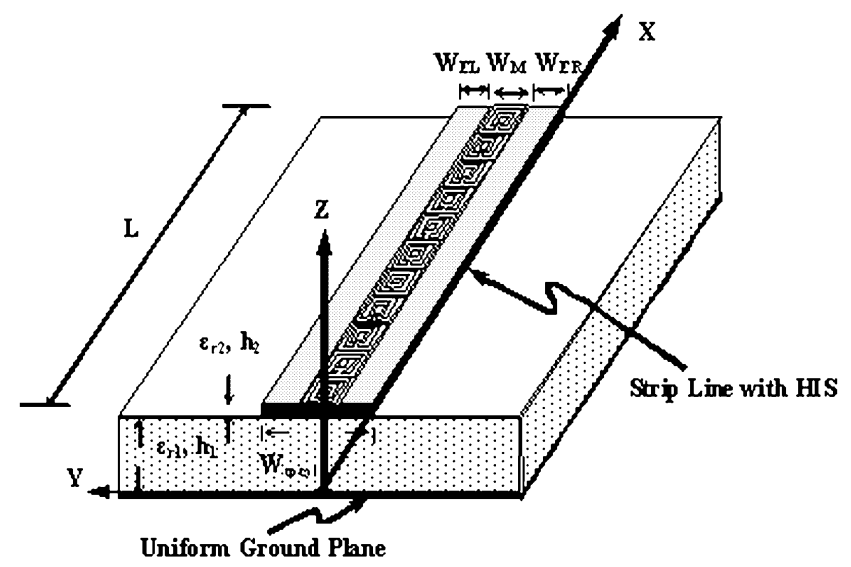

(a)

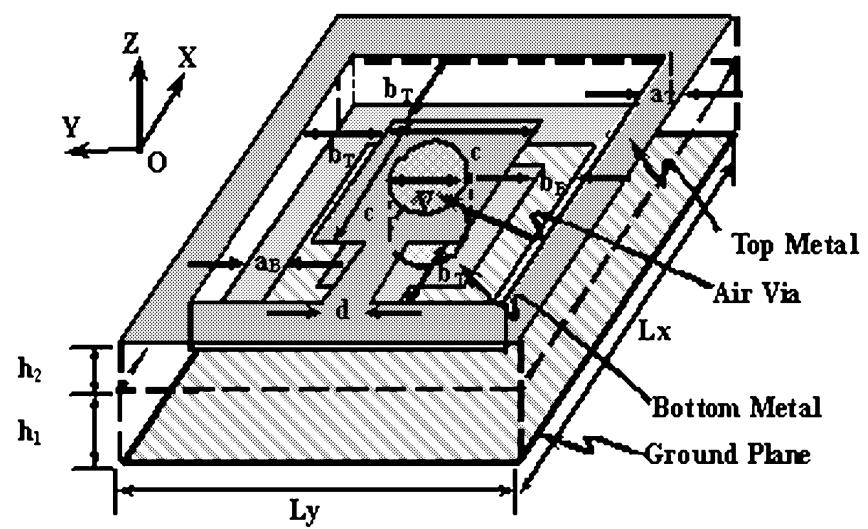

(b)

Fig. 8. Geometry of the microstrip line with metal strip replaced by EME surfaces. $h_{1}$ and $h_{2}$ are the thickness of the substrates. $h_{1}=0.508 \mathrm{~mm}$, $\varepsilon_{r 1}=3.38, h_{2}=0.203 \mathrm{~mm}, \varepsilon_{r 2}=3.38, W_{\mathrm{EL}}=W_{\mathrm{ER}}=1.067 \mathrm{~mm}$, $W_{M}=2.134 \mathrm{~mm}, L=32.01 \mathrm{~mm}, L_{x}=L_{y}=2.134 \mathrm{~mm}, a_{T}=0.127$ $\mathrm{mm}, b_{T}=0.508 \mathrm{~mm}, \mathrm{c}=0.864 \mathrm{~mm}, d=a_{B}=b_{B}=0.254 \mathrm{~mm}$, and $\mathrm{V}=0.406 \mathrm{~mm}$.

confirming that the complex modes of the particular EME guiding structure are also of $+j \alpha \pm \beta$-type. Between 9.4 and $10.0 \mathrm{GHz}$, only one wave component is extracted, representing a leaky wave exists in the forward direction. Fig. 10 shows the scattering parameters of the EME microstrip with a stopband in good agreement with what defined by the Brillouin diagram. Above $9.4 \mathrm{GHz}$, the upper bound stopband frequency, the RPA value rises again with peak frequency at $10.0 \mathrm{GHz}$, which corresponds to the normalized attenuation constant of 0.28 , implying that the leaky wave is more easily excited. When the operating frequency reaches $10.1 \mathrm{GHz}$, an additional leaky wave component in the backward direction emerge. When the operating frequency is increased further and between 10.1 and $10.4 \mathrm{GHz}$, a pair of leaky mode solutions are obtained. The inset of Fig. 9 shows that the attenuation constants of the leaky modes are symmetrical about $\alpha / k_{0}=0$. Notice that the relative current amplitude of the leaky mode component in the forward direction is approximately 19.4 times greater than that of the backward leaky mode. Above $10.4 \mathrm{GHz}, \beta_{0,0}^{+}$ and $\beta_{0,0}^{-}$emerge again with negligible attenuation constants. 


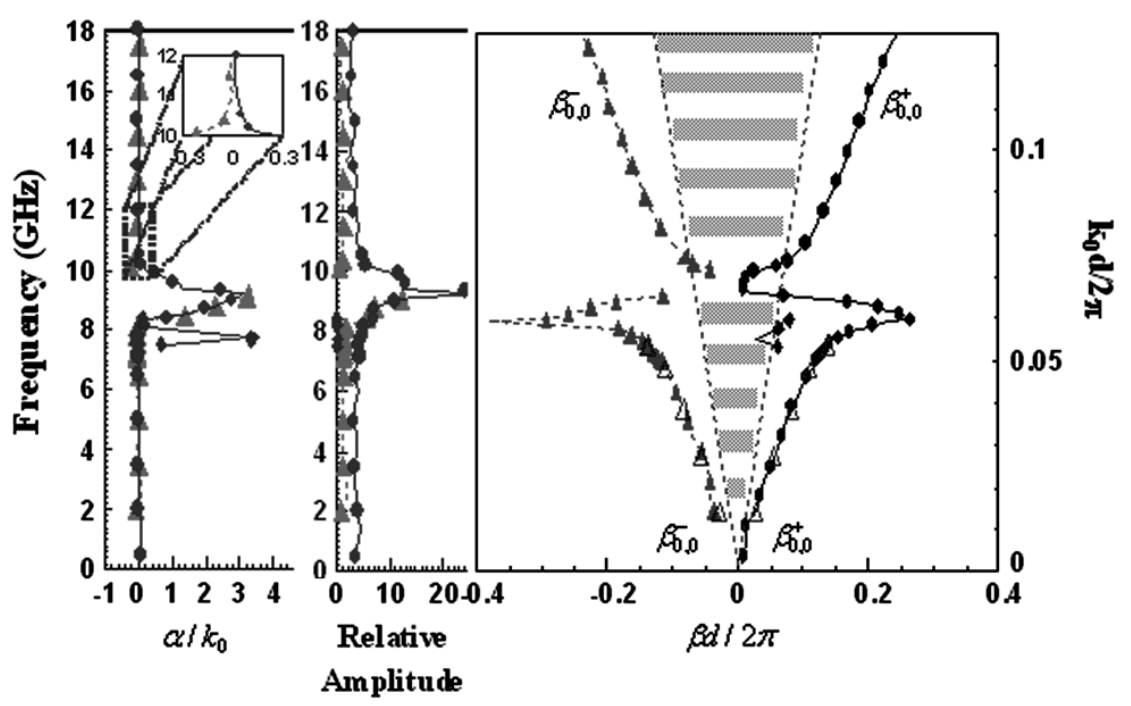

Fig. 9. Normalized attenuation constants, the relative current amplitudes, and the Brillouin diagram of the EME microstrip. The shadow region is labeled as the radiation region. The circles and the empty triangles, shown in the Brillouin diagram, represent the normalized phase constants obtained by invoking the matrix-pencil method and finite-element method, respectively. The solid line with solid circles and the dot lines with solid triangles represent the wave components in the forward and backward directions, respectively.

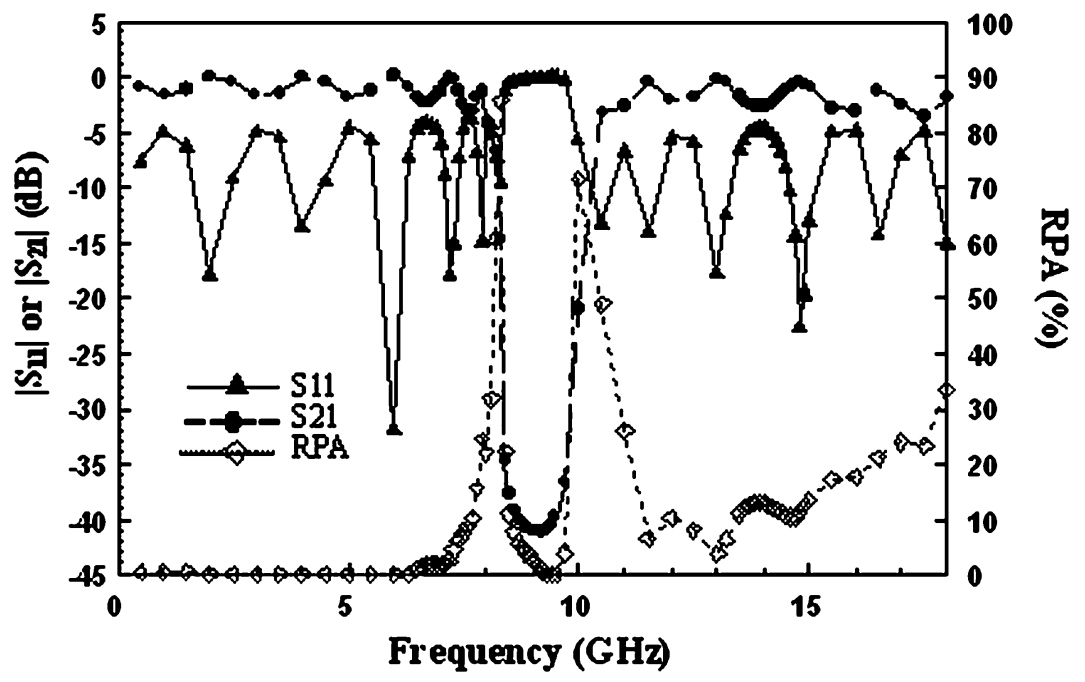

Fig. 10. Theoretical scattering parameters of the EME microstrip.

\section{SUMMARY AND CONCLUSION}

The matrix-pencil method demonstrates itself as an effective numerical procedure to extract the space harmonics (modal components) propagating in the periodical structures. Combining the matrix-pencil analyzes and the Brillouin diagram presentations illustrates the omnipotent ability to discover the unknown modal behaviors of the periodical structures and completely account for the results of the scattering analyzes. For the first time we may rigorously determine the boundary frequencies of the stopband of the complex periodical structures by observing the dispersion characteristics in the Brillouin diagram presentations.

The dispersion characteristics of three case studies, including microstrip with even-symmetric periodical perturbations, the microstrip on the EBG surface, and the EME microstrip, exhibit the different modal behaviors although the scattering analyzes showing the similar results such as the passband, stopband, and radiation regions. The matrix-pencil analyzes indicate that the complex mode pairs in the form of $+j \alpha \pm \beta$ or $-j \alpha \pm \beta$, which can carry purely reactive power in the guiding structure, exist in the stopband of the periodical structures. Around the corner frequencies of the stopband of the complex periodical structures such as the microstrip on the EBG structures and the EME microstrip, predominantly the space-wave leakage could be observed for the first time.

\section{ACKNOWLEDGMENT}

The authors wish to extend their sincere thanks to Professor Arthur A. Oliner for providing technical assistance and directing toward the $k-\beta$ diagram presentation of the periodical planar structure. 


\section{REFERENCES}

[1] W. S. C. Chang, "Periodic structures and their application in integrated optics," IEEE Trans. Microw. Theory Tech., vol. 21, pp. 775-785, Dec. 1973.

[2] S. T. Peng, T. Tamir, and T. L. Bertoni, "Theory of periodic dielectric waveguides," IEEE Trans. Microw. Theory Tech., vol. 23, pp. 123-133, Jan. 1975.

[3] A. A. Oliner and S. T. Peng, "New physical effects on periodically grooved open dielectric waveguides," Radio Sci., vol. 19, pp. $1251-1255,1984$

[4] R. E. Collin and F. J. Zucker, Antenna Theory. New York: McGrawHill, 1969.

[5] L. Brillouin, Wave Propagation in Periodic Structures. New York, NY: Dover, 1953.

[6] A. A. Oliner, "Radiating periodic structures: Analysis in terms of k vs. b diagrams," Short Course on Microwave Field and Network Techniques, Jun. 4, 1963.

[7] — "Periodic structures and photonic-band-gap terminology: Historical perspectives," in Proc. 29th Eur. Microwave Conf., Munich, Germany, Oct. 4-8, 1999, pp. 295-298.

[8] R. Coccioli, F. R. Yang, K. P. Ma, and T. Itoh, "Aperture-coupled patch antenna on UC-PBG substrate," IEEE Trans. Microw. Theory Tech., vol. 47, pp. 2123-2130, Dec. 1999.

[9] F. R. Yang, K. P. Ma, Y. Qian, and T. Itoh, "A uniplanar compact photonic-bandgap (UC-PBG) structure and its applications for microwave circuits," IEEE Trans. Microw. Theory Tech., vol. 47, pp. 1509-1514, Aug. 1999.

[10] V. Radisic, Y. Qian, and T. Itoh, "Broad-band power amplifier using dielectric photonic bandgap structure," IEEE Microw. Guided Wave Lett., vol. 8, pp. 13-14, Jan. 1998.

[11] H. Y. D. Yang, N. G. Alexopoulos, and E. Yablonovitch, "Photonic bandgap materials for high-gain printed circuits antennas," IEEE Trans. Antennas Propag., vol. 45, pp. 185-187, Jan. 1997.

[12] J. D. Shumpert, W. J. Chappell, and L. P. B. Katehi, "Parallel-plate mode reduction in conductor-backed slots using electromagnetic bandgap substrates," IEEE Trans. Microw. Theory Tech., vol. 47, pp. 2099-2104, Dec. 1999.

[13] T. K. Wu, Ed., Frequency Selective Surface and Grid Array. New York: Wiley, 1995.

[14] D. Sievenpiper, L. Zhang, R. F. J. Broas, N. G. Alexopolous, and E. Yablonovitch, "High-impedance electromagnetic surfaces with a forbidden frequency band," IEEE Trans. Microw. Theory Tech., vol. 47, pp. 2059-2074, Nov. 1999.

[15] M. J. Hill, R. W. Ziolkowski, and J. Papapolymerou, “A high-Q reconfigurable planar EBG cavity resonator," IEEE Microw. Guided Wave Lett., vol. 11, pp. 255-257, Jun. 2001.

[16] C. K. Wu, H. S. Wu, and C. K. Tzuang, "Electric-magnetic-electric (EME) slow-wave microstrip line and bandpass filter of compressed size," IEEE Trans. Microwave Theory Tech., vol. 50, pp. 1996-2004, Aug. 2002.

[17] C. K. Wu and C. K. Tzuang, "Slow-wave propagation of microstrip consisting of electric-magnetic-electric (EME) composite metal strips," in IEEE MTT-S Int. Microwave Symp. Dig., Phoenix, AZ, May 20-25, 2001, pp. 727-730.

[18] Y. C. Chen, C. K. Wu, and C. K. Tzuang, "Dual-frequency electricmagnetic-electric microstrip leaky-mode antenna of s single fan beam," IEEE Trans. Microw. Theory Tech., vol. 50, pp. 2713-2720, Dec. 2002.

[19] C. K. Wu and C. K. Tzuang, "Dispersion characteristics of EME microstrip at first higher order," in IEEE MTT-S Int. Microwave Symp. Dig., Seattle, WA, Jun. 2-7, 2002, pp. 1083-1086.

[20] S. T. Peng and R. B. Hwang, "Analysis of two-dimensionally periodic structures graphical methods and physical consequences," in Proc. 29th Eur. Microwave Conf., Munich, Germany, Oct. 4-8, 1999, pp. 299-302.

[21] P. S. Kildal and A. Kishk, "EM modeling of surfaces with STOP or GO characteristics-Artificial magnetic conductors and soft and hard surfaces," Appl. Comput. Electromagn. Soc. J., vol. 18, no. 1, pp. 32-40, Mar. 2003.

[22] Y. C. Chen and C. K. Tzuang, "Dispersion characteristics of microstrip on uniplanar compact photonic-bandgap (UC-PBG) ground plane and electric-magnetic-electric (EME) microstrips," in Proc. Asia-Pacific Microwave Conf. (APMC 2001), vol. 2, Taipei, Taiwan, R.O.C., Dec. 3-6, 2001, pp. 779-782.
[23] C. K. Tzuang and J. M. Lin, "On the mode-coupling formation of complex modes in a nonreciprocal finline," IEEE Trans. Microw. Theory Tech., vol. 41, pp. 1400-1408, Aug. 1993.

[24] J. R. Pierce, "Coupling of modes of propagation," J. Appl. Phys., vol. 25, no. 2, pp. 179-183, Feb. 1954.

[25] K. C. Chen, Y. Qian, C. K. Tzuang, and T. Itoh, "A periodic microstrip radial antenna array with a conical beam," IEEE Trans. Antennas Propag., vol. 51, pp. 756-765, Apr. 2003.

[26] C. K. Tzuang and Y. C. Chen, "Dispersion characteristics of microstrip with periodic perturbations," in IEEE MTT-S Int. Microwave Symp. Dig., Boston, MA, Jun. 11-16, 2000, pp. 1537-1540.

[27] R. S. Adve, T. K. Sarkar, O. M. C. Pereira-Filho, and S. M. Rao, "Extrapolation of time-domain responses from three-dimensional conducting objects utilizing the matrix pencil technique," IEEE Trans. Antennas Propag., vol. 45, pp. 147-156, Jan. 1997.

[28] A. S. Omar and K. Schunemann, "The effect of complex modes at finline discontinuities," IEEE Trans. Microw. Theory Tech., vol. 34, pp. 1508-1514, Dec. 1986.

[29] W. X. Huang and T. Itoh, "Complex modes in lossless shielded microstrip lines," IEEE Trans. Microw. Theory Tech., vol. 36, pp. 163-165, Jan. 1988.

[30] K. F. Fuh and C. K. Tzuang, "The effects of covering on complex wave propagation in gyromagnetic slotlines," IEEE Trans. Microw. Theory Tech., vol. 43, pp. 1100-1105, May 1995.

[31] G. J. Chou and C. K. Tzuang, "Oscillator-type active-integrated antenna: The leaky-mode approach," IEEE Trans. Microw. Theory Tech., vol. 44, pp. 2265-2272, Dec. 1996.

[32] H. S. Wu, C. K. Tzuang, and K. C. Chen, "Simultaneous suppression and enhancement of crosstalk of microstrip discontinuities by periodical lattice structures," in Proc. Int. Signal. Syst., Electron. Symp., Tokyo, Japan, Jul. 24-27, 2001, pp. 730-737.

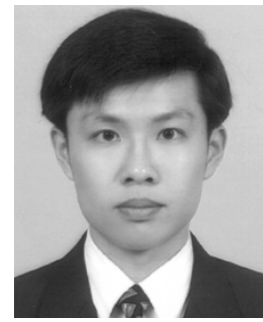

Yu-Chiao Chen (S'99-M'04) was born in Tainan, Taiwan, R.O.C., on June 25, 1975. He received the B.S., M.S., and Ph.D. degrees in communication engineering from the National Chiao Tung University, Hsinchu, Taiwan, R.O.C., in 1998, 2000, and 2003 , respectively.

His current research interests include field theory analysis and application designing of periodical structures.

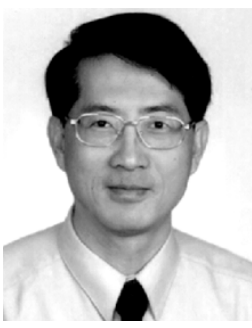

Ching-Kuang C. Tzuang (S'80-M'80-SM'92 -F'99) received the B.S. degree in electronic engineering from the National Chiao Tung University, Hsinchu, Taiwan, R.O.C., in 1977, the M.S. degree from the University of California at Los Angeles, in 1980, and the Ph.D. degree in electrical engineering from the University of Texas at Austin, in 1986.

From 1981 to 1984, he was with TRW, Redondo Beach, CA, where he was involved with analog and digital MMICs. Since 1986, he has been with the Institute of Communication Engineering, National Chiao Tung University. In February 2004, he joined the Graduate Institute of Communication Engineering, Department of Electrical Engineering, National Taiwan University, Taipei, Taiwan. His research activities involve the design and development of millimeter-wave and microwave active and passive circuits and the field theory analysis and design of various complex waveguiding structures and large-array antennas. He has supervised 61 M.S. students and 20 Ph.D. students.

Dr. Tzuang helped in the formation of the IEEE Microwave Theory and Techniques Society (IEEE MTT-S) Taipei chapter, and served as Secretary, Vice Chairman, and Chairman in 1988, 1989, and 1990, respectively. He has been on the Asia-Pacific Microwave Conference International Steering Committee, where, since 1994, he has represented the Taipei chapter as the International Liaison Officer. 


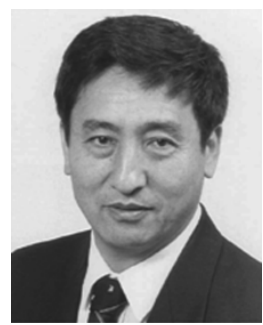

Tatsuo Itoh (S'69-M'69-SM'74-F'82) received the $\mathrm{Ph} . \mathrm{D}$. degree in electrical engineering from the University of Illinois, Urbana-Champaign, in 1969.

From September 1966 to April 1976, he was with the Electrical Engineering Department, University of Illinois. From April 1976 to August 1977, he was a Senior Research Engineer in the Radio Physics Laboratory, SRI International, Menlo Park, CA. From August 1977 to Jun. 1978, he was an Associate Professor at the University of Kentucky, Lexington. In July 1978, he joined the Faculty at The University of Texas at Austin, where he became a Professor of electrical engineering in 1981 and Director of the Electrical Engineering Research Laboratory in 1984. During summer 1979, he was a Guest Researcher at AEG-Telefunken, Ulm, West Germany. In September 1983, he became Hayden Head Centennial Professor of Engineering at The University of Texas. In September 1984, he was appointed Associate Chairman for Research and Planning of the Electrical and Computer Engineering Department, The University of Texas. In January 1991, he joined the University of California, Los Angeles, as Professor of electrical engineering and holder of the TRW Endowed Chair in Microwave and Millimeter Wave Electronics. He was an Honorary Visiting Professor at Nanjing Institute of Technology, China, and at the Japan Defense Academy. In April 1994, he was appointed Adjunct Research Officer for the Communications Research Laboratory, Ministry of Post and Telecommunication, Japan. He currently holds a Visiting Professorship at the University of Leeds, U.K. He has 310 journal publications and 640 refereed conference presentations. He has written 30 books/book chapters in the area of microwaves, millimeter-waves, antennas, and numerical electromagnetics. He has generated $60 \mathrm{Ph} . \mathrm{D}$. students.

Dr. Itoh is a Member of the Institute of Electronics and Communication Engineers of Japan, and Commissions B and D of USNC/URSI. He was elected a Member of the National Academy of Engineering in 2003. He has received a number of awards, including the Shida Award from the Japanese Ministry of Post and Telecommunications in 1998, Japan Microwave Prize in 1998, IEEE Third Millennium Medal in 2000, and IEEE MTT Distinguished Educator Award in 2000. He was Chairman of USNC/URSI Commission D from 1988 to 1990 and Chairman of Commission D of the International URSI for 1993 to 1996. He is Chair of the Long Range Planning Committee of URSI. He serves on advisory boards and committees of a number of organizations. He was Editor of IEEE TRANSACTIONS ON MiCROWAVE THEORY AND TEChNiQues for 1983-1985. He was Vice President of the Microwave Theory and Techniques Society in 1989 and President in 1990. He was elected an Honorary Life Member of the MTT Society in 1994. He was Editor-in-Chief of IEEE MiCROWAVE AND GUIDED WAVE LETTERS from 1991 through 1994. $\mathrm{He}$ is on the Administrative Committee of IEEE Microwave Theory and Techniques Society. He serves on advisory boards and committees for a number of organizations.

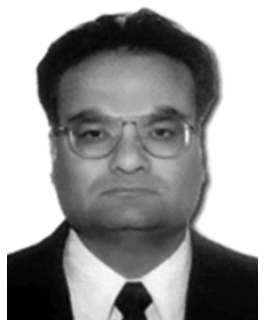

Tapan Kumar Sarkar (S'69-M'76-SM'81-F'92) received the B.Tech. degree from the Indian Institute of Technology, Kharagpur, India, in 1969, the M.Sc.E. degree from the University of New Brunswick, Fredericton, NB, Canada, in 1971, and the M.S. and Ph.D. degrees from Syracuse University, Syracuse, NY, in 1975.

From 1975 to 1976 , he was with the TACO Division of General Instruments Corporation. He was with the Rochester Institute of Technology, Rochester, NY, from 1976 to 1985 . He was a Research Fellow at the Gordon McKay Laboratory, Harvard University, Cambridge, MA, from 1977 to 1978 . He is now a Professor in the Department of Electrical Engineering and Computer Science, Syracuse University. He has authored or coauthored more than 250 journal articles, numerous conference papers, chapters in books, and several books including his most recent, Iterative and Self Adaptive Finite-Elements in Electromagnetic Modeling (Boston, MA: Artech House, 1998), Wavelet Applications in Electromagnetics and Signal Processing(Boston, MA: Artech House, 2002), and Smart Antennas (New York: Wiley, 2003). He is on the editorial board of J. of Electromagnetic Waves and Applications and Microwave and Optical Technology Letters. His current research interests deal with numerical solutions of operator equations arising in electromagnetics and signal processing with application to system design.

Dr. Sarkar is a Registered Professional Engineer in the State of New York. He is a Member of Sigma Xi and the International Union of Radio Science (URSI) Commissions A and B. He received the College of Engineering Research Award in 1996 and the Chancellor's Citation for Excellence in Research from Syracuse University in 1998. He obtained one of the "Best Solution" awards at the Rome Air Development Center (RADC) Spectral Estimation Workshop in May 1977, and received the Best Paper Award from the National Radar Conference in 1997 and the IEEE TRANSACTIONS ON ELECTROMAGNETIC COMPATIBILITY in 1979. He received the title Docteur Honoris Causa from Universite Blaise Pascal, Clermont Ferrand, France, and from the Politechnic University of Madrid, Madrid, Spain, in 1998 and 2004, respectively. He received the medal of the Friend of the City of Clermont Ferrand, France, in 2000. He was the Chairman of the Inter-commission Working Group of International URSI on Time Domain Metrology from 1990 to 1996. He was an Associate Editor for feature articles of the IEEE Antennas and Propagation Society Newsletter from 1986 to 1988. He was a Distinguished Lecturer for the Antennas and Propagation Society from 2000 to 2003. He is currently a Member of the IEEE Electromagnetics Award Board and an Associate Editor for the IEEE TRANSACTIONS ON ANTENNAS AND PROPAgation. He is the Vice President of the Applied Computational Electromagnetics Society (ACES) and the technical Chair for the combined IEEE 2005 Wireless Conference along with ACES to be held in Hawaii. 